\title{
Interest Point Detection in Video Surveillance Image Based on Topology Structure Features of Directed and Weight Complex Network
}

\author{
Qingyu Zou ${ }^{1, a^{*}}$, Baisheng Liu ${ }^{1, b}$, Jianwen Guan ${ }^{1, c}$ and Yan Chen ${ }^{1, d}$ \\ ${ }^{1}$ College of Electrical and Information Engineering of Beihua University, Jilin, China

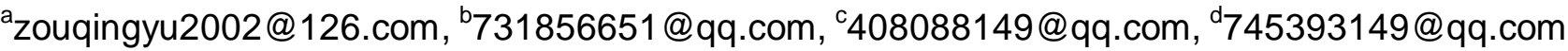

*The corresponding author

Keywords: Complex network; Image; Degree; Betweenness; Interest point

\begin{abstract}
Extracting and describing the key information of images are the focus of the image research. Interest point detection is an important part of image target recognition process. This paper presents a new method for detecting interest points in digital images. In this paper, the pixels in the image is taken as the network node, and the similarity and distance between the pixels are taken as the weight of the edge, and then the image is expressed as the directional and weighted network. We propose a new network node feature evaluation standard based on the integration of degree and betweenness. According to this standard, we extract the key nodes in the network, which are interest points of image. In order to verify the effectiveness of our algorithm, we use the algorithm in the complex video surveillance images. The results show that our algorithm could identify interest nodes from the images effectively.
\end{abstract}

\section{Introduction}

Simulating human visual ability is one of the main research directions of modern computer vision. Extracting and describing the key information of images are the focus of the image research. Key points as the important objects for image local feature extraction have been get more and more attention. At present, the definition of key points in the academic circles has not been unified. For different key detection methods, the key points may mean different. In general, the key point is the point where the brightness of the image changes drastically or the curvature maxima on the edge curve of the image. The ultimate goal of key detection is to describe the key points of information to achieve the image matching between each other to achieve image stitching, face recognition, video tracking and other applications. Moravec proposed a method of using gray-scale variance to detect key points in 1977, but this method does not have rotational invariance. In 1988, Harris and Stephens proposed a Harris corner detection algorithm based on the differential operation and the autocorrelation matrix to detect the key points. Lowe proposed an efficient SIFT key detection algorithm, which is a milestone in the field of local feature research[1]. Bay proposed the SNR key detection algorithm, which further improves the feature extraction speed and feature description performance.

Complex network is an important research object in the field of complex system., The theory of complex network has been paid more and more attention by scholars and successfully applied in many scientific fields since the discovery of small world and scale-free[2, 3]. It establish the model of complex network use the mathematical graph theory, only consider the topological relations between the nodes and other topological relation. Therefore, if we can use the complex network as a description model of the plane image, then this model will be able to effectively adapt to the image shape changes. Sun presents a scale-invariant approach to detect the interest points of images using complex network analysis[4]. Criado introduced a new method for detecting interest points in digital images making use of Complex Network Analysis. They proposed a general post-processing localization method based on centrality measures on a weighted version of the line-graph $L(G)$ after the association of a spatial and weighted complex network $G$ to each image with a prescribed geometrical structure[5].

In this paper, we introduce a novel approach to computing the interest points of an image using complex network analysis. We construct a weighted and directed complex network model to represent 
each image that gives some valuable information about the location of the interest points. The nodes in the network model are the pixels of the image and the edge is the relationship between the pixels, according to variations of the intensity, color and other parameters. Then the interest points could been identified through the topology of the image network model.

\section{Network Model of Image}

The description of the image as a complex network model is based on the complex network theory for image recognition of the premise and foundation. The complex network model is divided into unqualified network, undirected weight network, directed network and directed weight network.

We use the directed and weighted network to describe the image. Super pixel is a region-based image segmentation approach and aims to represent images with a limited number of super pixels which are clusters of neighboring real pixels in an image[6]. The super pixels in the image are taken as the nodes of the network, and the luminance difference $\left|I\left(p_{i}\right)-I\left(p_{j}\right)\right|$ of the two super pixels $[i, j]$ is used as the weight between the two points $w_{i, j}$, when the image is grayscale. When the image is a color map, the edge weight $w_{i, j}$ between node $i$ and $j$ is the RGB European distance of them.

$$
W_{i, j}=\frac{\sqrt{\left(R_{i}-R_{j}\right)^{2}+\left(G_{i}-G_{j}\right)^{2}+\left(B_{i}-B_{j}\right)^{2}}}{\sqrt{3}}
$$

Where R, G, and B are the RGB values of the pixels, respectively. The direction of the edge in the network is the brightness of the pixels point to the brightness of small pixels.

In order to make the uniform network into a complex network, we calculate the Euclidean distance between any two pixels. Then set the radius threshold $r$ and delete the links between the nodes in the network where the distance are greater than $\mathrm{r}$. The final weight of the complex network links $w_{i j}$ is given by the formula (2)[5, 7-9]. The complex network model of the image is constructed as shown in Fig. 1. The characteristics of the complex network model of image are shown in the Table 1.

$$
w_{i j}=\left\{\begin{array}{l}
\left|I\left(p_{i}\right)-I\left(p_{j}\right)\right|, \quad\left(\operatorname{dist}\left(p_{i}, p_{j}\right) \leq r\right) \\
0, \quad\left(\operatorname{dist}\left(p_{i}, p_{j}\right)>r\right)
\end{array}\right.
$$
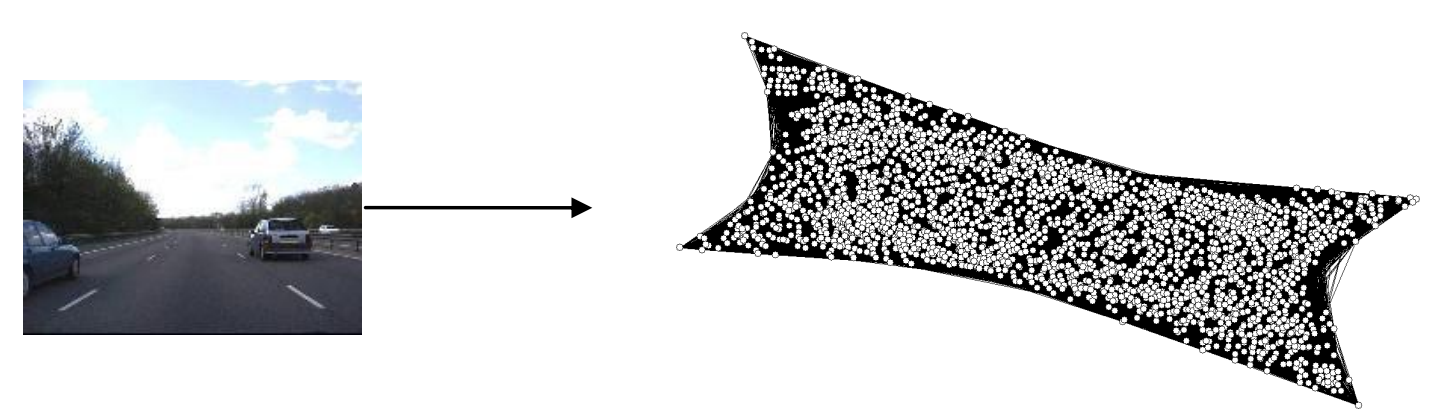

Figure 1. Complex network model of image. The picture has been done using the software Pajek[10]

\section{Interest point detection}

We measure the characteristics of the network node according to the topology of the complex network, after constructing the complex network model of the image. And then according to the structural characteristics of the node to identify the key nodes of the network, which is the interest point of image. The feature of node $i$ is $\zeta_{i}=\alpha D e g_{i}+(1-\alpha) B e n_{i}$, where $D e g_{i}$ is the outdegree of node $i$. Ben is the betweenness of node $i$. Degree use as a centrality measure, although degree centrality is a simple centrality measure[11]. In directed networks, nodes have both an in-degree and an out-degree, and both may be useful as measures of centrality in the appropriate circumstances. The degree of a node in a 
network is the sum of weight of all the links connected to it. For image network models, there are an out-degree $k_{\text {out }}$ and an in-degree $k_{\text {in }}$ which are, respectively, the sum of regulating and regulated relations at the node $k_{i}^{\text {out }}=\sum_{j=1}^{n} A_{i j}, \quad k_{j}^{i n}=\sum_{i=1}^{n} A_{i j}$. Where $A$ is the adjacency matrix of network, $A_{i j}$ is an element of matrix $A, A_{i j}=1$ if there is a link from node $i$ to node $j$. The betweenness centrality of a node $i$ is defined to be the number of the shortest paths that pass through $i$. It express the extent to a node lies on the shortest paths between other nodes. Nodes with high betweenness centrality may have considerable influence within a network by virtue of their control over information passing between others. In a directed network the shortest path between two vertices depends, in general, on the direction you travel in. The shortest path from A to B is different from the shortest path from B to A. Indeed there may be a path in one direction and no path at all in the other. Thus it is important in a directed network explicitly to include the path counts in either direction between each vertex pair. Mathematically, let $n_{j k}^{i}$ be 1 if node $i$ lies on the shortest path from $j$ to $k$ and 0 if it does not. Then the betweenness $B_{i}$ is given by $B_{i}=\sum_{j \neq k} n_{j k}^{i}$.

\section{Results and Discussion}

In order to verify the effectiveness of the proposed algorithm, we use this method for video monitoring image. Fig. 2 shows the results produced by our interest point detection algorithm. This method based on degree and betweenness centrality was applied by filtering the best $5 \%-10 \%$ of the points with highest interest, producing 100 interest points. Using this method can be obtained accurately identify the interest points of the vehicle target in the image. Fig. 3 shows the results produced by Harris interest point detection algorithm. Fig. 4 shows the results produced by SIFT interest point detection algorithm.

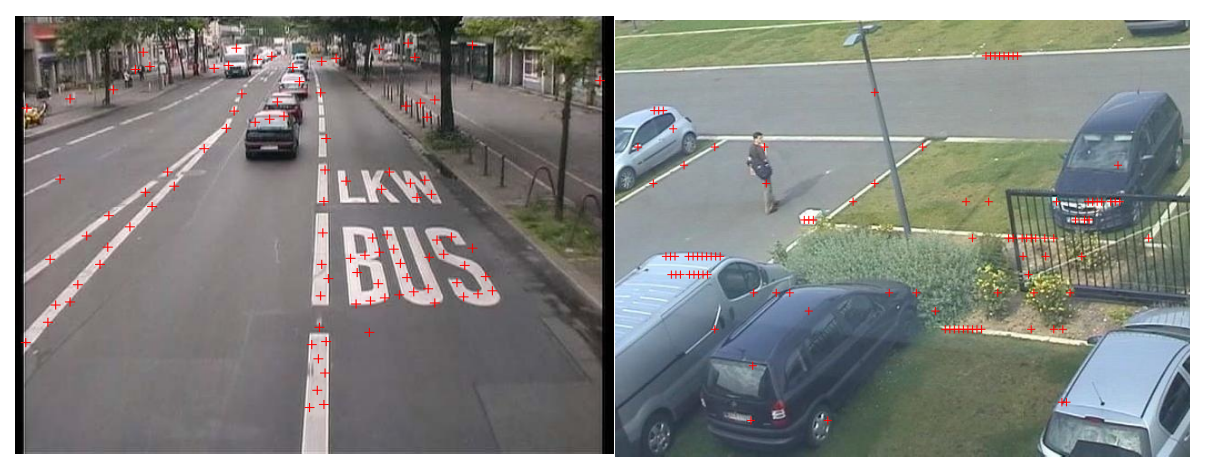

Figure 2. The interest point identify results of our algorithm

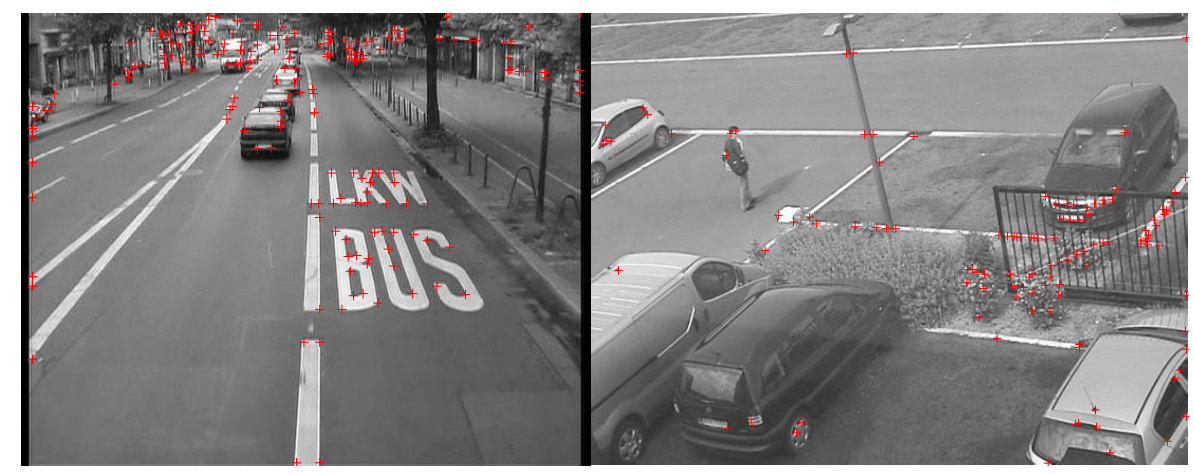

Figure 3. The interest point identify results of Harris algorithm 


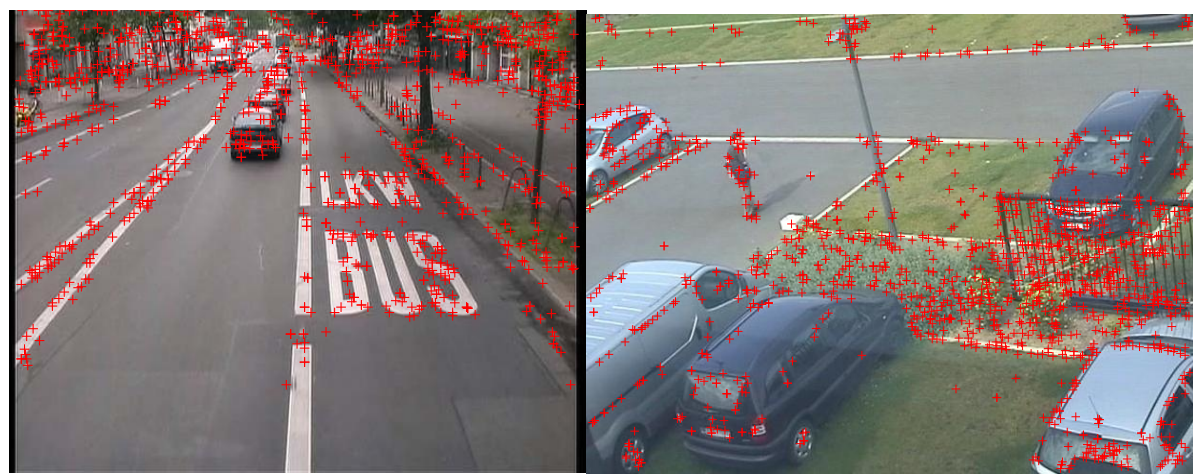

Figure 4. The interest point identify results of SIFT algorithm

The empirical tests show that the results obtained by three methods in the same picture are quite different. We compare in two different test images the interest point detect results achieved by our complex network based algorithm with Harris and SIFT algorithm. We measured the effectiveness of five results produced by these algorithms using the ratio of the effective nodes to the identified nodes. The results as shown in Table 1. In a certain sense, our algorithm improves the efficiency and accuracy of image target recognition.

Table 1 The effectiveness of identify results

\begin{tabular}{|c|c|c|c|}
\hline Algorithm & $\begin{array}{c}\text { Effectiveness } \\
\text { of left image }\end{array}$ & $\begin{array}{c}\text { Effectiveness } \\
\text { of right image }\end{array}$ & $\begin{array}{c}\text { Used for color } \\
\text { pictures directly }\end{array}$ \\
\hline Our & $89 \%$ & $77 \%$ & Yes \\
\hline Harris & $58.9 \%$ & $93 \%$ & No \\
\hline SIFT & $48.7 \%$ & $58 \%$ & Yes \\
\hline
\end{tabular}

\section{Conclusion}

This paper presents a new interest point detection algorithm in images based on complex network. Firstly, the complex network model of image is constructed based on the characteristics of image pixels. Then identify the interest nodes according to two topological features of image complex network model, which are degree and betweenness. The experimental results show that our methods is more efficient and accurate for the recognition of interest nodes of image objects compared with the traditional method.

\section{Acknowledgements}

This work is supported mostly by the Jilin Province department of education Science and Technology Plan Projects (Grant NO 201658), partially by the Beihua University Dr. Scientific Research Fund (Grant NO. 2015557).

\section{References}

[1] C. D., R. V., Real-time tracking of non-rigid objects using mean shift Computer Vision and Pattern Recognition. 2 (2000) 142-9.

[2] A. L. Barabasi, Network science, Philosophical Transactions of the Royal Society a-Mathematical Physical and Engineering Sciences. 371 (2013) 1-3.

[3] A. L. Barabasi, NETWORK SCIENCE Luck or reason, Nature. 489 (2012) 507-8.

[4] X. Sun, K. Zhao, Z. Lu, et al., Scale-invariant interest point detection in images based on complex network analysis, ICIC Express Letters, Part B: Applications. 7 (2016) 525-30.

[5] R. Criado, M. Romance, A. Sanchez, A post-processing method for interest point location in 
images by using weighted line-graph complex networks, International Journal of Bifurcation and Chaos in Applied Sciences and Engineering. 22 (2012) 1250163.

[6] O. Cuadros, G. Botelho, F. Rodrigues, et al. Segmentation of large images with complex networks. 25th Conference on Graphics, Patterns and Images, SIBGRAPI 2012, August 22, 2012 - August 25, 2012. Ouro Preto, Brazil: IEEE Computer Society; 2012. p. 24-31.

[7] J. d. A. S. Wesley Nunes Gonçalves, Odemir Martinez Bruno. A Rotation Invariant Face Recognition Method Based on Complex Network. In: Isabelle Bloch RMCJ, editor. 15th Iberoamerican Congress on Pattern Recognition. Sao Paulo2010. p. 426-33.

[8] A. R. Backes, O. M. Bruno, Shape classification using complex network and Multi-scale Fractal Dimension, Pattern Recognition Letters. 31 (2010) 44-51.

[9] R. Criado, M. Romance, A. Sanchez, Interest point detection in images using complex network analysis, Journal of Computational and Applied Mathematics. 236 (2012) 2975-80.

[10] W. d. Nooy, A. Mrvar, V. Batagelj, Exploratory social network analysis with Pajek, Cambridge University Press, New York, 2011.

[11] M.E.J.Newmen, Networks: An Introduction, Oxford University Press, New York, 2010. 